\title{
Interference and auditory short-term memory in the bottlenosed dolphin*
}

\author{
LOUIS M. HERMAN \\ University of Hawaii, Honolulu, Hawaii 96822
}

\begin{abstract}
Interference in auditory short-term memory in the bottlenosed dolphin, Tursiops truncatus (Montagu), was studied using a delayed matching-to-sample task. At each trial, one of two sample sounds, chosen randomly, was projected underwater for 4 sec and then, after a variable delay interval, both sounds were presented. A response to the sound matching the initial sample was reinforced. Correct matching was significantly reduced following short intervals between trials in combination with long delays after the sample (proactive interference), or when a near continuous irrelevant sound was inserted into the delay interval (retroactive interference). There was rapid habituation to interference if the irrelevant sound was short in duration relative to the delay interval. For both proactive and retroactive interference, the errors were predominantly responses to the sample sound appropriate to the prior trial rather than to the current trial, indicating that memory for the relative recency of events (temporal memory) was degraded by interference. When interference was deleted or minimized, temporal memory remained nearly perfect over 30 -sec delay intervals, the longest tested. The importance of distinguishing between temporal memory and nontemporal, or event, memory in different forms of the delayed matching task was emphasized.
\end{abstract}

The delayed matching-to-sample task has been used to test animal short-term memory in a number of recent studies (see reviews of D'Amato, 1973; Jarrard \& Moise, 1971). In this task, a sample item occurs for a fixed duration, and then, after a variable delay interval, two or more test items are presented. A response to the test item matching the sample is reinforced.

In auditory delayed matching tests, the bottlenosed dolphin shows little forgetting of once-presented, novel auditory items over unfilled delay intervals as long as $120 \mathrm{sec}$ (Herman \& Gordon, 1974). During the unfilled intervals, no stimulation was given and no responses were required. In visual delayed matching tests using a small set of visual items repeated in random sequence over trials, capuchin monkeys also demonstrate excellent matching capabilities after $120-\mathrm{sec}$, or longer, unfilled delays (e.g., D'Amato \& Worsham, 1972), while Carneaux pigeons respond at almost chance levels after unfilled delays of only $10 \mathrm{sec}$ or less (e.g., Berryman, Cumming, \& Nevin, 1963; Smith, 1967). The data for dolphin and monkey together provide little encouragement for decay theories of animal short-term memory, which postulate the rapid, spontaneous weakening with time of memory for an item.

Support for interference theories of animal short-term memory has been found in additional studies of delayed matching in monkeys and pigeons, showing that retention of the current sample item decreases with increased recency of the prior sample (proactive interference) (Jarrard \& Moise, 1971, Fig. 9), or if

\footnotetext{
*This research was supported by Grant GB-32148X from the National Science Foundation and is Contribution No. 452 from the Hawaii Institute of Marine Biology. Requests for reprints should be sent to Louis M. Herman, Department of Psychology, University of Hawaii, Honolulu, Hawaii 96822.
}

irrelevant stimulation is given or responses are required during the delay interval (retroactive interference) (Etkin, 1972; Moise, 1970; Worsham \& D'Amato, 1973; Zentall, 1973).

In any task involving a delay of response, a correct response may depend on the animal's ability to remember the relative recency of the events of the current and preceding trials (e.g., Winograd, 1971), and D'Amato (1973) has interpreted delayed matching as principally a temporal discrimination problem. When dealing with a small set of sample items in repeated use, correct matching on a given trial requires that the animal remember which was the most recently presented sample. Increases in the delay intervals following samples and conditions of interference can impair the retention of relative recency and thereby increase the matching error. D'Amato assumes that monkeys are better at making and maintaining temporal discriminations than are pigeons, accounting for their differences in delayed matching performance.

In the present experiments, the effects of interference on auditory delayed matching were studied in the bottlenosed dolphin. In contrast to the unlimited set of novel sounds used as samples by Herman and Gordon (1974), only two sounds were used in the present study. One or the other sound, chosen randomly, was the sample on a given trial and both were test sounds following the delay interval. Experiment $\mathbf{I}$, on proactive interference, examined effects of the length of the intertrial interval (ITI), the duration of the delay interval, and whether the current-trial sample was the same as, or different from, the prior-trial sample. Since samples were repeated over trials, matching responses could potentially be controlled by the prior-trial sample 
if there was difficulty in remembering the relative recency of samples.

Experiment II, on retroactive interference, studied effects of the type, locus, and amount of irrelevant auditory stimulation given during the delay interval, as well as continuing the investigation of control of responding by successive samples. Besides providing basic data on the effects of interference in an animal previously showing very little auditory short-term forgetting with interference minimized (Herman \& Gordon, 1974), the two experiments were directed to the further evaluation of the issue of temporal discrimination in delayed matching.

\section{GENERAL METHOD}

\section{Subject and Apparatus}

The $S$ was an adult female bottlenosed dolphin, Tursiops truncatus (Montagu), named Keakiko, used previously by Herman and Gordon (1974). The daily diet was $7 \mathrm{~kg}$ of whole smelt. These approximately 120 fish were almost all consumed during testing sessions.

The 15.2-m-diam circular testing tank and the underwater apparatus (slightly modified) were as described in Herman and Gordon (1974). Briefly, two pairs of vertically suspended ropes formed the left and right sides of an $0.9-\mathrm{m}$-wide $\times 0.5-\mathrm{m}$-long underwater channel leading to a start paddle. The region between the channel exit and the 1-m distant start paddle was the listening area, the focal region for two Cheseapeake $J 9$ underwater speakers that projected the sample and test sounds. The two speakers were $90 \mathrm{deg}$ left and right of the start paddle at $1.8 \mathrm{-m}$ distance and slightly to the side and front of each was a response paddle.

A University MM-2PPS underwater speaker was positioned $1 \mathrm{~m}$ beyond the start paddle and projected sounds for controlling entry into the channel, exit from the listening area, and indicating to the animal that the response paddle selected was correct. The retroactive interference sounds (irrelevant stimuli) also were projected from this speaker.

The sound-generation equipment was described previously (Herman \& Arbeit, 1973; Herman \& Gordon, 1974); measurements of underwater signal levels and of noise characteristics of the testing tank are available in those sources.

\section{Training}

The animal was trained over a 3-week period to match either of two sample sounds without bias, with unfilled delay intervals of 1 to $10 \mathrm{sec}$ and ITIs of 20 to $60 \mathrm{sec}$. Bias was defined as a tendency to respond to only one of the two test sounds, regardless of the sample sound. Three different pairs of sounds were used successively during this training. The first pair, used for 236 trials, yield ed $21.2 \%$ matching errors; $82.5 \%$ of these were biased responses, the animal persistently responding to one test sound. For the second pair, given for 726 trials, matching errors increased only to $23.3 \%$, but biased responding increased to $93.5 \%$.

To guard against the further development of biased responding, an error-correction procedure was used with the third sound pair, and the sample duration was increased from 2.5 to 4 sec. During 64 trials with this pair, there were only three matching errors ( $4.7 \%$ error), two within the first 10 trials. Consequently, this third sound pair, the 4-sec sample duration, and the correction procedure (described subsequently) were continued in the following experiments. The sound pair consisted of: (a) a $7.5-\mathrm{kHz}$ pulsed pure tone, with pulse rate of $13 \mathrm{~Hz}$ and duty cycle of $50 \%$, and (b) a frequency-modulated sound with center frequency of $3 \mathrm{kHz}$, the frequency varying sinusoidally $\pm 10 \%$ about this center at a rate of $3 \mathrm{~Hz}$. Sound pairs of this general type, a pulsed tone vs a frequency-modulated sound with a different primary frequency, are easily discriminated by the bottlenosed dolphin (Herman \& Arbeit, 1973).

\section{General Procedure}

Each trial began with a 10-sec maximum duration sound from the University speaker, controlling entry into the listening area through the underwater channel. A push on the start paddle ended the sound and was followed, sequentially, by a 2-sec pause; the 4-sec sample sound projected simultaneously from the two J9 speakers; the delay interval; and the two test sounds, projected from the $\mathrm{J} 9$ speakers in sequential order, for $2.5 \mathrm{sec}$ each, separated by a $.5-\sec$ silent interval. One test sound occurred at each $\mathrm{J} 9$, the matching sound randomly appearing at the left or right $\mathrm{J} 9$. Also, the matching sound was randomly first or second in the sequence. A response on the paddle adjacent to the $\mathbf{J} 9$ that projected the matching sound was reinforced. If scheduled, an irrelevant sound was projected from the University speaker during the delay interval.

Exiting from the listening area to press a paddle was controlled by a continuous sound from the University speaker, beginning $.5 \mathrm{sec}$ after the two test sounds ended. A paddle response ended the exit sound and played back the selected test sound from the $\mathrm{J} 9$ for $2.5 \mathrm{sec}$. If the playback was the matching sound, it was followed by a .5 -sec sound from the University speaker and a thrown fish reward. The ITI began .5 sec after playback terminated.

The chain described maintained the animal within the listening area during stimulus presentation and also controlled the ITI duration. Playback was previously shown necessary for the acquisition of two-choice auditory discriminations, with sequentially presented sounds (Herman \& Arbeit, 1973). The control sound characteristics and the contingencies following inappropriate responding within the chain were described elsewhere (Herman \& Arbeit, 1973; Herman \& Gordon, 1974).

On correction trials, the sample sound of the preceding error trial was repeated, but the location of the matching test sound at the left or right $\mathrm{J} 9$ speaker and the sequential order of matching and nonmatching test sounds were each randomly varied from that of the error trial. The predetermined sequence of sample sounds resumed after a response to the matching sound on a correction trial or after a maximum of four unsuccessful corrections.

\section{EXPERIMENT I: PROACTIVE INTERFERENCE}

The variables were ITI duration and the length of the delay following the sample. The experiment was conducted in three parts, each testing a different set of IT I values.

\section{Procedure}

In Part A, the ITIs were 15, 30, and 60 sec; in Part B, 7.5 and $15 \mathrm{sec}$; and in Part C, 7.5 and $30 \mathrm{sec}$. The 7.5-sec value was a lower practical limit, since approximately that much time was required for the animal to retrieve and eat its fish reinforcement following a correct response and then return to the entrance to the underwater channel. The same four delays between sample and test sounds-1, 5,15 , and 30 sec-were used in all parts of the experiment.

There were generally two daily testing sessions. Sessions were divided into three blocks of 16 trials in Part $A$ and into two blocks of 24 trials in Parts B and C, each block within a session 
testing a different ITI. There were $2 \mathrm{~h}$ between daily sessions and 30 min between blocks within sessions.

Part A consisted of 13 sessions. The order of testing of the three ITIs across blocks was counterbalanced in blocks of six. In Part B, there were 12 sessions and in Part C, 21 sessions; in each case, the two ITIs were tested in counterbalanced sequence every four blocks.

Within blocks, all variables were balanced. Each of the two sounds served as the sample on a random half of the trials, the maximum run length held to four, and was followed equally often by each delay interval. Each delay interval occurred once every four trials, in an order governed by 4 by 4 Latin squares. Following the delay, the test sound matching the sample was first in the sequence of two test sounds on half the trials, last on the remainder, and was projected equally often from the left-hand and right-hand $\mathrm{j} 9 \mathrm{~s}$.

\section{Results and Discussion}

Decreasing the ITI below $30 \mathrm{sec}$ decreased the percentage of correct responses in all three parts of the experiment (Fig. 1). In Part A, th ough the 30- and 60 -sec ITIs each produced near errorless performance, the ITI main effect was highly significant $(F=6.94$, $\mathrm{df}=2 / 36, \mathrm{p}<.01$ ). A Newman-Keuls test revealed that performance after the 15 -sec ITI was significantly poorer $(p<.01)$ than after either of the two longer ITIs. ITI was also highly significant in Part $C(F=22.7, d f=1 / 40$, $\mathrm{p}<.001)$, but failed significance in Part $\mathrm{B}(\mathrm{F}=3.03$, $\mathrm{df}=1 / 22, \mathrm{p}<.10)$. Together, the three parts show that matching was significantly poorer with ITIs of $15 \mathrm{sec}$ or less than with IT Is of $30 \mathrm{sec}$ or greater.

These ITI effects were generally consistent with findings on visual delayed matching in macaque monkeys, except for a shift in the temporal break-point for interference (Jarrard \& Moise, 1971, Fig. 9). Matching accuracy of the monkeys was significantly reduced at 5-sec ITIs relative to 15-, 30-, and 60-sec ITIs, while there was little difference in effects of the latter three intervals.

Delay was not significant in Part A $(F=2.24$, df $=3 / 108, p<.10$ ), primarily because of the near errorless performance at all delays during the 30- and 60-sec ITIs, but was significant during Parts $\mathrm{B}(\mathrm{F}=3.38$, $\mathrm{df}=3 / 66, \quad \mathrm{p}<.05)$ and $\mathrm{C} \quad(\mathrm{F} 11.7, \mathrm{df}=3 / 120$, $\mathrm{p}<.001)$. The mean number of correct responses at 1 and 5 -sec delays in Parts $B$ and $C$ each significantly exceeded $(p<.05)$ the mean number of correct responses at 15- and 30-sec delays, but other comparisons were not significant (Newman-Keuls tests). There was no significant interaction between ITI and delay in any part $(\mathrm{F}<1.0$ in each case).

The near errorless performance after all delays with ITIs of 30 and $60 \mathrm{sec}$ in Part A confirmed results of Herman and Gordon (1974) that, with interference minimized, delay alone may not be sufficient to cause losses in auditory short-term memory in the bottlenosed dolphin. It is not clear whether the somewhat reduced performance during replication of the $30-\mathrm{sec}$ ITI in Part $\mathrm{C}$ was due to a gradual buildup of proactive interference over the successive parts of the experiment or to the particular combination of ITIs tested.

Errors associated with successive two-trial events (digrams) were tallied by sessions of 48 trials, yielding 47 digrams per session, except for one session in Part B terminated early because of equipment malfunction. Since there were no errors on the first trial of any session, all errors were accounted for. Table 1 shows that the error rate was considerably higher when the sample sound was changed across successive trials than when it

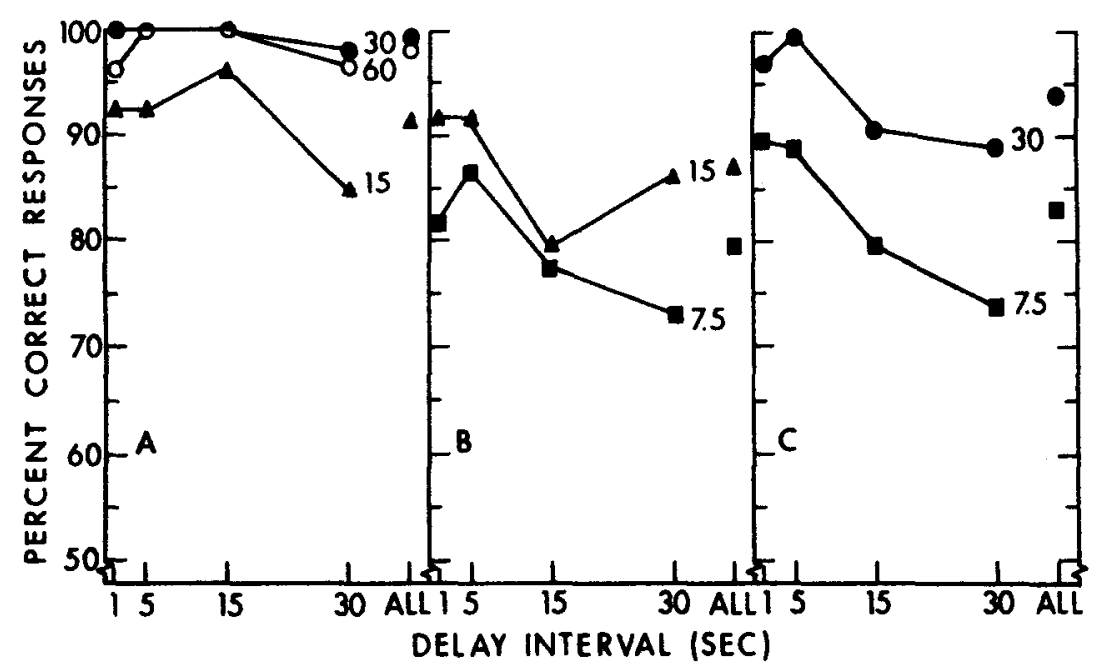

Fig. 1. Results for Parts A, B, and C of Experiment I, showing the percentage of correct responses at each delay interval between sample and test sounds, as a function of the listed ITI duration: $7.5,15,30$, or 60 sec. The overall percentage of correct responses at each ITI is shown by the isolated data point at the right of each graph. 
Table 1

The Probability of an Error After Changing the Sample Sound from the Prior Trial and After Not Changing

\begin{tabular}{cccccc}
\hline & \multicolumn{2}{c}{ Changed Sample } & \multicolumn{2}{c}{ Same Sample } & Percent \\
\cline { 5 - 6 } $\begin{array}{c}\text { Experi- } \\
\text { ment }\end{array}$ & $\begin{array}{c}\text { Number } \\
\text { Trials }\end{array}$ & $\begin{array}{c}\text { Percent } \\
\text { Error }\end{array}$ & $\begin{array}{c}\text { Number } \\
\text { Trials }\end{array}$ & $\begin{array}{c}\text { Percent } \\
\text { Error }\end{array}$ & $\begin{array}{c}\text { Changed } \\
\text { Trials }\end{array}$ \\
\hline Ia & 370 & 5.4 & 241 & 1.2 & 60.6 \\
Ib & $298^{*}$ & 30.2 & $233^{*}$ & 0.4 & 56.1 \\
Ic & 670 & 16.4 & 317 & 2.2 & 67.9 \\
Combined & 1338 & 16.4 & 791 & 1.4 & 62.8 \\
Ila & 134 & 3.7 & 54 & 3.7 & 71.3 \\
IIb & 345 & 15.4 & 219 & 4.6 & 61.2 \\
Combined & 479 & 11.1 & 273 & 4.4 & 63.7 \\
\hline
\end{tabular}

*Total of 33 trials omitted in both columns combined.

was unchanged (Part A, $\chi^{2}=6.57, p<.02 ;$ Parts $B$ and $\left.\mathrm{C}, \chi^{2} \geqslant 37.4, p<.001\right)$.

Since the average probability of a sample change from trial to trial was .63 , reflecting the limitations on run length of a sample sound, the trial sequences actually favored the switching of responses from trial to trial. The greater error rate on changed trials shows, however, that an alternation strategy did not develop, indicating that the animal was not controlled by this characteristic of the trial sequences (cf. D'Amato \& O'Neill, 1970).

The markedly greater error rate after a change in the sample sound across trials and the significantly reduced matching accuracy with ITIs of $15 \mathrm{sec}$ or less seem interpretable as failures in temporal discrimination, a difficulty in remembering which was the last sample sound heard (cf. D’Amato, 1973; Winograd, 1971). Shorter ITIs decrease the temporal interval between the current and the prior sample, reducing the temporal discriminability of these events and increasing the error probability if the sample changes across trials.

With the short ITIs in Parts B and C, significantly fewer correct matches occurred after delays of 15 and $30 \mathrm{sec}$ than after delays of 1 or $5 \mathrm{sec}$. This finding may also be interpreted as a failure in temporal discrimination. As delays increase, the temporal interval between the test sounds and the current-trial sample becomes more like the interval between these same test sounds and the prior-trial sample (the ratio of the shorter interval to the longer interval increases with increased delays), yielding an increased probability of responding to the memory for the prior-trial sample. It would be valuable to compare the present sequential-trial effects with findings for monkeys and pigeons, since these effects bear directly on the temporal discrimination hypothesis (D'Amato, 1973). However, digram analyses of errors in delayed matching appear not to have been made for these other species.

\section{EXPERIMENT II: RETROACTIVE INTERFERENCE}

The effects of auditory stimulation during the delay interval were studied, using two different irrelevant sounds. Part A varied the temporal point of interpolation within the delay interval of a short irrelevant sound while Part B studied the effects of a near continuous irrelevant sound.

\section{Procedure}

The irrelevant sounds were $1-\mathrm{kHz}$ and $10-\mathrm{kHz}$ pure tones, bracketing the lower $(3 \mathrm{kHz})$ and upper $(7.5 \mathrm{kHz})$ limits of the primary frequencies used for the two sample sounds. The ITI was maintained at $30 \mathrm{sec}$, since little proactive interference was found with this ITI in Experiment I. Sessions, two per day, consisted of two blocks of 24 trials separated by a 30-min interval.

In Part A, there was a total of four sessions. The irrelevant sounds occurred only during Sessions 2 and 3, Sessions 1 and 4 being controls. At Session 1, the delay interval was $1 \mathrm{sec}$ throughout the first 24-trial block and $15 \mathrm{sec}$ during the second 24-trial block, while at Session 4 these delay values were reversed across blocks. At Sessions 2 and 3, the delay was always $15 \mathrm{sec}$, during which a single irrelevant sound of 4-sec duration was presented, beginning either $3 \mathrm{sec}$ after (early interpolation) or $8 \mathrm{sec}$ after (late interpolation) the start of the delay.

During each 24-trial block, the balancing of variables was as in Experiment I, Parts B and C. In addition, there was complete balancing of the pairing of each irrelevant sound with each sample sound and of each irrelevant sound with each of the two temporal points of interpolation.

In Part B there were 12 sessions. During one 24-trial block of a session, an irrelevant sound occurred during each delay interval, while for the remaining block, the delays were unfilled. The ordering of these two conditions across blocks was counterbalanced every four blocks. The balancing of other variables was in Part $\mathrm{A}$.

The delay interval was $15 \mathrm{sec}$ throughout Part B and the irrelevant sound, of 13-sec duration, was centered in the delay interval. However, during the first block having irrelevant sounds, their duration was increased gradually over the first ten trials from an initial value of $4 \mathrm{sec}$ to the final value of $13 \mathrm{sec}$, and during the second block with ir relevant sounds, the same fade-in schedule was completed in five trials. Thereafter, the duration was always $13 \mathrm{sec}$. This procedure provided a gradual transition from the 4-sec duration used for ir relevant sounds in Part A.

\section{Results and Discussion}

In Part $A$, the two control sessions (no interference sounds) produced only two errors, one at Session 1 at a 1 -sec delay and one at Session 4 at a 15 -sec delay. There were five errors at the first interference session, four during the first 24-trial block and one during the second 24-trial block. There were no errors during the second interference session. This rapid habituation to the presence of the irrelevant sounds made further testing of these interference conditions unwarranted. Overall, there were $89.6 \%$ correct responses during interference sessions and $95.8 \%$ correct responses at control sessions.

Of the five errors during interference sessions, four followed early interpolation $(p=.16)$ and three were associated with the $10-\mathrm{kHz}$ pure tone $(p=.31)$. The few errors precluded any firm evaluation of trends.

Figure 2 shows results for Part B. At each session, there was poorer performance during the block with irrelevant (interference) sounds than during the paired 


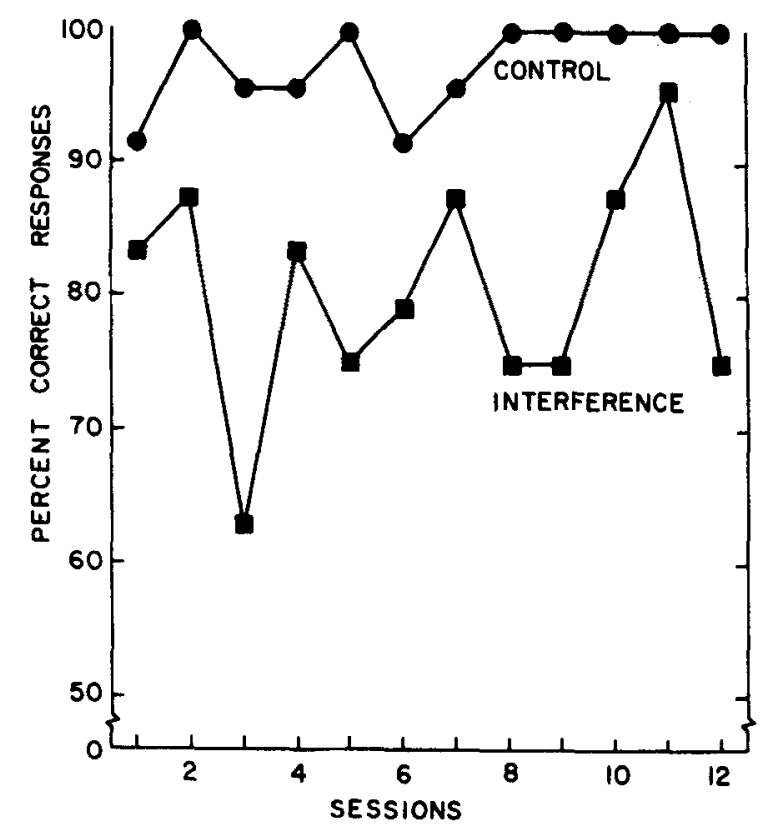

Fig. 2. Experiment IIB. The percentage of correct responses at each session during the control (no-interference) block of 24 trials and during the interference block of 24 trials.

control (no-interference) block (sign test, $\mathrm{p}=.00025$ ). In contrast to Part A, habituation to the irrelevant sounds did not occur. Aside from Sessions 3 and 11, matching performance during interference blocks remained between $75.0 \%$ and $87.5 \%$ correct. There were $80.6 \%$ correct responses overall during interference blocks and $97.6 \%$ correct responses overall during control blocks.

Of the total of 56 errors during interference blocks, $66.1 \%$ followed the $10-\mathrm{kHz}$ interference tone $\left(\chi^{2}=6.1\right.$, $\mathrm{p}<.05)$. The $1-$ and $10 \mathrm{kHz}$ tones were projected at equal sound pressure levels, but because of the relatively greater hearing sensitivity of the bottlenosed dolphin at $10 \mathrm{kHz}$ (Johnson, 1968), it is probable that the high frequency tone was perceived as louder. The fact that it was more interfering may reflect the greater attention or distraction value of louder sounds.

As in Experiment 1 , the error rate in Part B was significantly higher $\left(\chi^{2}=13.9, \mathrm{p}<.001\right)$ following a change in the sample sound across trials than following unchanged samples (Table 1). In Part A, error rates were the same following changed and unchanged samples, but this was based on a total of only seven errors.

Clearly, irrelevant sounds can significantly impair matching performance if they are long relative to the delay. The temporal point of interpolation, a variable possible only with short irrelevant sounds, was ineffective under the procedures used. Thus, the amount of interference during the delay was more important than was the location of the interference within the delay. That the $10-\mathrm{kHz}$ irrelevant sound was more detrimental than the $1-\mathrm{kHz}$ sound suggests that an additional variable of importance can be the type of interference.

These results were similar to some findings with monkeys. Moise (1970) found that performance of monkeys on visual delayed matching decreased significantly with increasing durations of the irrelevant stimuli. Also, Moise (1970) and Etkin (1972) found no significant differences between early and late interpolation of irrelevant visual stimuli, though either condition produced consistently poorer performance than a control (no-interference) condition.

\section{GENERAL DISCUSSION}

Proactive and retroactive interference markedly impaired auditory delayed matching performance in the bottlenosed dolphin. Data from the two experiments suggested that memory for the relative recency of events was degraded by the interference. There was an increased probability of responding to the sample sound appropriate to the prior trial, rather than to the current trial, when short ITIs were combined with long delays between sample and test sounds (proactive interference) or when there were near continuous irrelevant sounds during the delay interval (retroactive interference). Deletion of these interference conditions generally resulted in almost errorless performance over delays of up to $30 \mathrm{sec}$, the longest tested.

The proactive interference conditions were seen as directly decreasing the discriminability of the temporal intervals separating the current retention test from the current and prior samples (cf. D'Amato, 1973). It is less clear how retroactive interference reduces temporal discriminability. Any explanation must account for the effects of retroactive interference type and duration, significant variables in the present study and in some visual delayed matching studies of monkeys and pigeons (Moise, 1970; Worsham \& D'Amato, 1973; Zentall, 1973), and of habituation to retroactive interference, observed in Experiment IIA of this study and also found in pigeons (Zentall, 1973). Those cases in which habituation to retroactive interference was not observed may reflect either an inadequate number of trials (e.g., in Experiment IIB of the present study, only 288 interference trials were given) or the presence of some reinforcement structure to support continued responding to the retroactive stimulation (e.g., Moise, 1970).

An attentional view can explain these retroactive-interference effects satisfactorily. In this view, it is assumed that retroactive interference distracts from (shifts attention from) the primary memory task of remembering the relative recency of samples, resulting in losses in recency information. The type of retroactive interference determines the probability of a shift in attention, while its duration influences the length of the shift. If attending to retroactive interference is not 
reinforced, habituation develops and performance eventually reaches the level of the no-interference control condition.

Worsham and D'Amato (1973) reported that attentional responses of their monkeys to recorded sounds of monkey vocalizations actually improved visual delayed matching performance at long delays, relative to a no-vocalization control. This paradoxical finding, in apparent contradiction to an attentional analysis, might simply reflect the control of disruptive emotional responding by this type of stimulation. D'Amato (1973) and Herman and Gordon (1974) have observed negative emotional responding in the monkey and the dolphin, respectively, during long unfilled delay intervals and have noted the correlation of these emotional responses with poor matching performance. For the monkeys studied by Worsham and D'Amato (1973), the aversive properties of the long delays might have been reduced by positive conditioned emotional responses to the recorded monkey vocalizations.

Finally, but importantly, caution is necessary in viewing delayed matching as exclusively a temporal discrimination problem (cf. D'Amato, 1973). In this study and in the cited monkey and pigeon delayed matching studies, a limited number of sample stimuli was presented repeatedly over trials, requiring the discrimination and retention of their temporal order. If an unlimited number of novel samples is used instead (e.g., Herman \& Gordon, 1974), no sample from a prior trial ever appears again and the discrimination of the temporal order of samples is no longer required. When using novel samples, memory for the attributes of once-presented items is tested, call it event memory, and the temporal location of the items is irrelevant. When using a limited sample set, memory for the temporal location of well-learned (repeated) items is tested, call it temporal memory, and the attributes of the items are considered permanently stored in memory.

Temporal memory in dolphins (present study) and in monkeys (e.g., D Amato \& Worsham, 1972) seem very well developed, in comparison with capacities of pigeons (Berryman, Cumming, \& Nevin, 1963; Smith, 1967), th ough interference to temporal memory appears to operate the same way across these different species (Etkin, 1970; Moise, 1970; Zentall, 1973). Event memory in the dolphin is also well developed (Herman \&
Gordon, 1974), and work underway in our laboratory by $\mathrm{R}$. Thompson indicates that cynomolgus monkeys have excellent event memory, as measured in delayed matching tests using novel visual stimuli. Event memory, using a delayed matching task, has not been studied in the pigeon, and interference effects in event memory have not yet been examined for any species.

Our experience has been that training for event memory in monkeys and dolphins is considerably simpler than is training for temporal memory, and that delay intervals may be more easily stretched in event memory. Perhaps this reflects the relative susceptibility of these different categories of memory to interference.

\section{REFERENCES}

Berryman, R., Cumming, W. W., \& Nevin, J. A. Acquisition of delayed matching in the pigeon. Journal of the Experimental Analysis of Behavior, 1963, 6, 101-107.

D'Amato, M. R. Delayed matching and short-term memory in monkeys. In G. H. Bower (Ed.), The psychology of learning and motivation: Advances in research and theory. Vol. 7. New York: Academic Press, 1973.

D'Amato, M. R., \& O'Neill, W. Matching behavior: Some methodological problems. Behavior Research Methods \& Instrum entation, 1970, 2, 162-164.

D'Amato, M. $\bar{R}$., \& Worsham, R. W. Delayed matching in the capuchin monkey with brief sample durations. Learning \& Motivation, 1972, 3, 304-312.

Etkin, M. W. Light produced interference in a delayed matching test with capuchin monkeys. Learning \& Motivation, 1972, 3 , 313-324.

Herman, L. M., \& Arbeit, W. R. Stimulus control and auditory diserimination learning sets in the bottlenose dolphin. Journal of the Experimental Analysis of Behavior, 1973, 19, 379-394.

Herman, L. M., \& Gordon, J. A. Auditory delayed matching in the bottlenose dolphin. Jowrnal of the Experimental Analysis of Behavior, 1974, 21, 19-26.

Jarrard, L. E., \& Mo ise, S. L. Short-term memory in the monkey. In L. E. Jarrard (Ed.), Cognitive processes of nonhuman primates. New York: Academic Press, 1971.

Johnson, C. S. Relation between absolute threshold and duration-of-tone pulses in the bottlenose porpoise. Journal of the Acoustical Society of America, 1968, 43, 757-763.

Moise, S. L. Short-term retention in Macaca speciosa following interpolated activity during delayed matching from sample. Journal of Comparative \& Physiological Psychology, 1970, 73, 506-514.

Smith, L. Delayed discrimination and delayed matching in pigeons. Journal of the Experimental Analysis of Behavior, $1967,10,529-533$.

Winograd, $\mathbf{E}$. Some issues relating animal memory to human memory. In W. K. Honig and P. H. R. James (Eds.), Animal memory. New York: Academic Press, 1971.

Worsham, . W., \& D'Amato, M. R. Ambient light, white noise and monkey vocalization as sources of interference in visual short-term memory in monkeys. Journal of Experimenta Psychology, 1973, 99, 99-105.

Zentall, T. R. Memory in the pigeon: Retroactive inhibition in a delayed matching task. Bulletin of Psychonomic Society, 1973, 1, 126-128.

(Received for publication April 19, 1974; revision accepted August 8,1974 .) 